2018-03

Insulin resistance is higher in prepubertal girls but switches to become higher in boys at age 16: A Cohort Study (EarlyBird 57)

Jeffery, SC

http://hdl.handle.net/10026.1/11187

10.1111/pedi.12571

Pediatric Diabetes

Wiley

All content in PEARL is protected by copyright law. Author manuscripts are made available in accordance with publisher policies. Please cite only the published version using the details provided on the item record or document. In the absence of an open licence (e.g. Creative Commons), permissions for further reuse of content should be sought from the publisher or author. 


\title{
Insulin resistance is higher in prepubertal girls but switches to become higher in boys at age 16: A Cohort Study (EarlyBird 57)
}

\author{
Sarah C Jeffery ${ }^{1,2}$ | Joanne Hosking ${ }^{1}$ | Alison N Jeffery ${ }^{1}$ (] | Michael J Murphy ${ }^{3}$ | \\ Linda D Voss ${ }^{1}$ | Terence J Wilkin ${ }^{4}$ | Jonathan Pinkney ${ }^{1}$
}

${ }^{1}$ Centre for Clinical Trials and Population Studies, Plymouth University Peninsula Schools of Medicine and Dentistry, Plymouth, UK

${ }^{2}$ Newcastle University Medical School, Newcastle, UK

${ }^{3}$ Division of Pathology and Neuroscience, University of Dundee, Dundee, UK

${ }^{4}$ Division of Endocrinology and Metabolism, Exeter Medical School, University of Exeter, Exeter, UK

\section{Correspondence}

Jonathan Pinkney, MD, FRCP, Centre for Clinical Trials and Population Studies,

Plymouth University and Peninsula Schools of Medicine and Dentistry, N6 Plymouth Science Park Phase 1, Plymouth, Devon PL6 8BX, UK. Email: jonathan.pinkney@plymouth.ac.uk

\begin{abstract}
Background: The risk of type 2 diabetes is increasing in teenage girls, and is associated with their greater insulin resistance (IR).

Hypothesis: We hypothesized that the adverse metabolic profile of girls (compared with boys) would persist from childhood through adolescence.

Patients and Methods: Community-based longitudinal cohort of 292 children (147 boys) studied annually from 9 to 16 years. Measures: IR (homeostasis-model-assessment-2), high-density lipoprotein-cholesterol (HDL-C), triglycerides, \% body-fat (dual-energy $x$-ray absorptiometry), pubertal stage (age at peak height velocity), physical activity (accelerometry). Multi-level modelling established the age-related trends in IR and lipids and the influence of covariates.

Results: Each year from 9 to 15 years, girls had $21 \%$ to $63 \%$ higher IR than boys (girls mean IR $0.73-1.33$, boys $0.51-0.89, P<.005)$. At 16 years the gender difference was not significant (girls IR 0.60 , boys $0.56, P=.45$ ). Girls had lower HDL-C from 9 to 12 years, higher triglycerides from 9 to 14 years, greater adiposity throughout, and earlier puberty, but boys were more active than girls (all $P<.05$ ).

After adjustment for \%-fat, puberty and activity, the gender difference in IR between girls and boys aged 9 to 15 years became non-significant (IR girls $0.66-1.01$, boys $0.65-1.04, P>.07$ ). However, after adjustment at 16 years, girls' IR was $25 \%$ lower than boys' (girls 0.44 , boys $0.63, P=.001)$, and they had $22 \%$ higher HDL-C $(P<.001)$ and $20 \%$ lower triglycerides $(P=.003)$.

Conclusions: The higher IR of prepubertal and early pubertal girls diminishes during late puberty, and boys begin to exhibit greater metabolic risk. Despite being leaner and more active, boys at 16 years have higher IR than girls, suggesting future higher risk for diabetes, thus we reject our hypothesis.
\end{abstract}

\section{KEYWORDS}

adiposity, adolescent, child, insulin resistance, physical activity

\section{1 | INTRODUCTION}

Type 2 diabetes (T2D) is presenting at increasingly younger ages, and the diagnosis is often made in the teenage years. ${ }^{1} \mathrm{~T} 2 \mathrm{D}$ in children has become a serious public health concern, and in some high-risk populations it is now more common than type 1 diabetes. $^{2}$ The risk of T2D in this age group is strongly associated with obesity ${ }^{2}$; $92 \%$ of children with T2D have been reported as overweight or obese. ${ }^{3}$

According to a large recent study from North America, T2D prevalence was 0.58 per 1000 in girls and 0.35 per 1000 in boys, ${ }^{4}$ and other studies have reported a female excess of T2D among children and adolescents. ${ }^{3,5,6}$ These observations contrast with findings in 
adults, where men are reported to be at higher risk than women. ${ }^{7,8}$ The explanation for the gender difference in the prevalence of T2D between adults and adolescents is uncertain, however, it has been proposed that endocrine changes leading to pubertal insulin resistance (IR) may be among the factors influencing the risk of T2D in children. ${ }^{9}$ There may be a "cross-over" of the gender difference in IR between childhood and adulthood, with a decline in IR in postpubertal females relative to males. These changes could reflect age- and gender-dependent changes in adiposity. Logue et $\mathrm{al}^{10}$ reported that men develop T2D at a lower body mass index (BMI) than women, and suggested this was because men are more insulin resistant and have more central fat than women.

Cross-sectional studies at birth ${ }^{11}$ and throughout the prepubertal years ${ }^{12,13}$ have consistently shown higher fasting insulin concentrations in females. These have also been observed in longitudinal studies during adolescence. ${ }^{14,15}$ The EarlyBird longitudinal study has previously reported higher levels of IR in 5-year-old girls compared with boys, even after accounting for differences in adiposity and physical activity. ${ }^{16}$ Moreover, it is well established that puberty is associated with a substantial increase in IR. ${ }^{17,18}$ Gender differences in IR have been attributed to differences in adiposity, fat distribution, sex hormones, and pubertal timing. ${ }^{19,20}$ The temporal influence of different factors can only be investigated with longitudinal studies. EarlyBird is a unique longitudinal study of the same children over a period of 12 years. Gender differences from 5 to 8 years have been reported previously. ${ }^{16}$ The aim of this analysis was to investigate gender differences in IR between 9 and 16 years. We hypothesized that the adverse metabolic profile of girls (compared with boys) would persist from childhood through adolescence.

\section{2 | PATIENTS AND METHODS}

\section{1 | Design, setting, and participants}

EarlyBird is a non-intervention longitudinal cohort study of 347 children (174 boys and 173 girls) recruited from 53 primary schools in Plymouth, UK. Exclusion criteria were diabetes, pathological states likely to affect growth or body composition, moderate or severe physical disability, and long-term use of oral steroids. A total of 307 children were recruited at 5 years in 2000 to 2001 and 40 more at age 9 years in 2004 to 2005 to redress a gender imbalance. Most were white Caucasian ( $n=342$ ) and 5 children ( 3 girls, 2 boys, 1.5\% of the cohort) were of mixed race, reflecting the racial mix of the city $(98.4 \%$ white Caucasian according to the 2001 census). ${ }^{21}$ Children were seen annually (mean interval 1.0 year, SD 0.1). Assent from the child at each visit and written consent from the parent were obtained. Ethical approval was granted by the Local Research Ethics Committee in 1999 and updated regularly. The study has been reported in detail elsewhere. ${ }^{22}$

\section{2 | Main outcome measure}

IR was measured by homeostasis model assessment-2 method using the Homeostasis Model Assessment of Insulin Resistance (HOMA-IR) program, downloaded from the University of Oxford Diabetes Trials
Unit website (www.dtu.ox.ac.uk). ${ }^{23}$ A venous blood sample was taken at 9 AM after an overnight fast. Serum insulin was measured by Immulite analyser (Diagnostic Products Corporation, Los Angeles, California), cross-reactivity with proinsulin $<1 \%$, inter-assay coefficient of variation (CV) $9 \%$, detection limit of the assay $2.0 \mathrm{mU} / \mathrm{L}(14 \mathrm{pmol} / \mathrm{L})$. Glucose was measured using Cobas Integra 700 analyser (Roche Diagnostics $\mathrm{GmbH}$, Mannheim, Germany; inter-assay CV 2\%) HOMA-IR has been validated against the euglycemic clamp $(r=0.91)$ in healthy youths. ${ }^{24}$

\section{3 | Secondary outcome measures}

High-density lipoprotein-cholesterol (HDL-C) and triglycerides were measured using Cobas Integra 700 analyser (Roche Diagnostics) and inter-assay CV $2 \%$.

\section{4 | Predictor variables}

Gender, age, height measured to nearest $1 \mathrm{~mm}$ (Leicester Height Measure, Child Growth Foundation, London),weight measured to nearest 200 g (Tanita Solar $1632 \mathrm{~W}$ electronic scales, West Drayton, London, UK), and BMI standard deviation scores (SDS) were calculated $^{25}$; waist circumference were measured by metal tape (Chasmors Ltd, London, UK); body fat (\% fat) was measured annually by dual energy $\mathrm{x}$-ray absorptiometry (DEXA) by Prodigy Advance fan beam densitometer (GE Healthcare, Chicago, Illinois, USA).

Pubertal status measured by age at peak height velocity (APHV), determined as the tangential velocity at the middle time-point of 3 consecutive height measurements taken 6 months apart. APHV, an objective measure of puberty, allows comparison of pubertal development between genders. ${ }^{26,27}$

Physical activity was measured by Actigraph accelerometers (Model 7164, Fort Walton Beach, Florida), having good technical reproducibility. ${ }^{28}$ Accelerometers were worn around the waist and recorded continuously for 7 days each year. The children wore the accelerometer every day from getting up until going to bed, removing it only for water-based activities, and keeping a diary of any activity undertaken while not wearing the accelerometer. Only recordings capturing at least 4 days of monitoring (each incorporating at least 9 hours' wear time, and including at least 1 weekend day) were included in the analyses. Time spent in moderate-and-vigorous intensity physical activities (MVPA; min/wk) was calculated against the threshold specific to 3 metabolic equivalents of task (METs), deemed by the UK Government to be the lower limit of moderate activity. ${ }^{29}$

Children's MVPA varied little from 9 to 16 years, ${ }^{30}$ and in order to characterize the overall long-term physical activity level of each child, their MVPA was averaged across all 8 time points. In doing so, the children were ranked according to their activity levels throughout the whole period with $95 \%$ reliability, compared with $70 \%$ reliability when using 1 annual measure of physical activity. ${ }^{30}$

\section{5 | Statistical analysis}

The distributions of HOMA-IR, triglycerides, \% fat, and MVPA, were skewed in both genders and were log-transformed for analysis and 
back-transformed for presentation. Cross-sectional analyses were performed using IBM SPSS version 22 (IBM Corp., Armonk, New York). The gender difference between mean values was expressed as a percentage. Differences between individuals were assessed by independent $t$ tests. Univariate regression explored the association between the outcome variable and predictor variables.

Longitudinal data were analyzed using linear mixed-effects modelling taking into account all available data at each time-point ( $R$ software ${ }^{31}$ using Imer function in Ime $4^{32}$ ). The effect of covariates on the gender difference in IR over time was examined. Random intercepts were included as well as age (categorized to allow for nonlinear change in IR over time), gender, \% fat, APHV, and MVPA as fixed effects. Two-way interactions between the covariates, age, and gender were examined and retained in the model if significant at the $5 \%$ level or of particular interest. Interpretation of the model coefficients was facilitated by plotting.

\section{3 | RESULTS}

A total of 292 children (147 boys) had measures of IR at 9 years ( $84 \%$ of the cohort), and these subjects were used for this analysis. Descriptive characteristics are shown in Table 1 . The girls attained peak height velocity 1.64 years before boys. Girls had significantly higher \% body fat at each age, larger waist circumferences from 10 years onwards, and a significantly higher BMlsds at 14 years $(P=.003)$.

IR rose and fell through puberty in both genders, with boys reaching peak IR at 14 years, and girls at 12 years. IR was significantly higher in girls than boys at each age up to 15 years, with the biggest gender difference at 12 years. Girls had $13 \%$ to $18 \%$ higher triglycerides than boys from 9 to 14 years $(P<.032)$, but the difference was no longer significant at 15 and 16 years. Boys had $3 \%$ to $7 \%$ higher HDL-C than girls from 9 to 12 years $(P<.02)$, whereas by 15 years girls had $7 \%$ higher HDL-C $(P=.02)$, and by 16 years girls were $9 \%$ higher $(P<.001)$.

\section{1 | Univariate regression}

Predictor variables were added separately at each age to assess their contribution to IR (Table S1, Supporting Information). The variable having the greatest effect on the gender difference in IR was \% fat. After adjusting for fat there was no significant gender difference in IR at ages 9 to 11,14 , and 15 years. At 12 and 13 years, girls were still more insulin resistant than boys after accounting for fat $(P<.001$ and .018 respectively), and at 16 years, girls were $40 \%$ less insulin resistant than boys $(P<.001)$. Waist circumference, BMI, APHV, and MVPA each had more modest effects in attenuating the gender difference in IR. When these variables were included in the models, girls remained more insulin resistant than boys throughout.

Adjusting for \% fat, APHV and MVPA increased the gender difference in HDL-C levels at 16 years, with \% fat having the greatest effect (girls $25 \%$ higher than boys; $P<.001$ ). The only predictor variable which influenced the gender difference in triglycerides at 16 years was \% fat, adjusting for which led to girls having $20 \%$ lower levels than boys $(P<.001)$.
Fasting glucose levels were significantly higher in the boys than girls at 10 years and 13 to 16 years. The gender difference in glucose was explained by APHV at 14 and 15 years, but adjusting for adiposity and MVPA made little difference.

\section{2 | Longitudinal modelling}

The measure of adiposity explaining the greatest proportion of the gender difference in IR was total \% fat, therefore this was included as a covariate in the longitudinal models along with objective measures of APHV and MVPA, as these are also known to influence IR.

Figure 1 shows mean (95\% confidence interval [CI]) IR in boys and girls from 9 to 16 years. Figure $1 \mathrm{~A}$ shows the unadjusted IR (model estimates are given in Table S2). Figure 1B shows IR adjusted for APHV alone, Figure $1 \mathrm{C}$ shows IR adjusted for APHV and MVPA, and Figure 1D shows IR adjusted for APHV, MVPA, and \% fat. The inclusion of all 3 covariates in the model affected the gender difference in IR such that there was a cross-over between 13 and 14 years so that by 16 years, IR in girls was approximately $25 \%$ lower than in boys.

Figure 2 shows the mean $(95 \% \mathrm{Cl}) \mathrm{HDL}-\mathrm{C}$ and triglycerides in boys and girls from 9 to 16 years. Figure 2A (raw data) shows that HDL-C fell from 9 to 16 years, with the fall more marked in boys. After adjustment (Figure 2B), HDL-C became significantly higher in the girls from 12 years onwards such that by 16 years, HDL-C was approximately $22 \%$ higher in girls than boys (Table S3). Triglycerides increased in both genders, girls having $13 \%$ to $18 \%$ higher levels than boys from 9 to 14 years (raw data, Figure 2C). However, after adjustment for \% fat, APHV and MVPA, there was no gender difference in triglycerides until 16 years when the girls had approximately $20 \%$ lower levels than boys (Figure 2D; Table S4).

\section{4 | DISCUSSION}

The principal finding of this study was that IR is significantly higher in adolescent girls than boys, and this gender difference narrows and reverses by age 16 years, at which age boys become more insulin resistant than girls. After adjusting for factors known to influence IR, the gender difference in IR between 9 and 15 years was largely attributable to differences in adiposity. Furthermore, these findings were largely mirrored by changes in levels of triglycerides and HDL-C levels, which support the likely biological significance of the temporal changes in IR.

Fasting glucose levels were slightly higher in boys, and this gender difference was attenuated to some extent by pubertal stage, and not by adiposity or activity. Relatively higher glucose in boys at 16 years (by $4 \%$ ) could have contributed to their higher adjusted IR at 16 years, but is unlikely to have affected their lipids.

This analysis extends the findings of Moran et al, ${ }^{14}$ who studied 350 children aged 11 to 15 years at baseline, with 55\% cohort retention at 18 to 19 years. In that study IR was higher in girls at 11 years, and higher in boys at 19 years. However, the authors did not find any association between pubertal development and IR. Our longitudinal dataset with 8 measurements at annual time points, 93\% cohort retention for measures of IR, and the small age range of the 
TABLE 1 Cohort characteristics

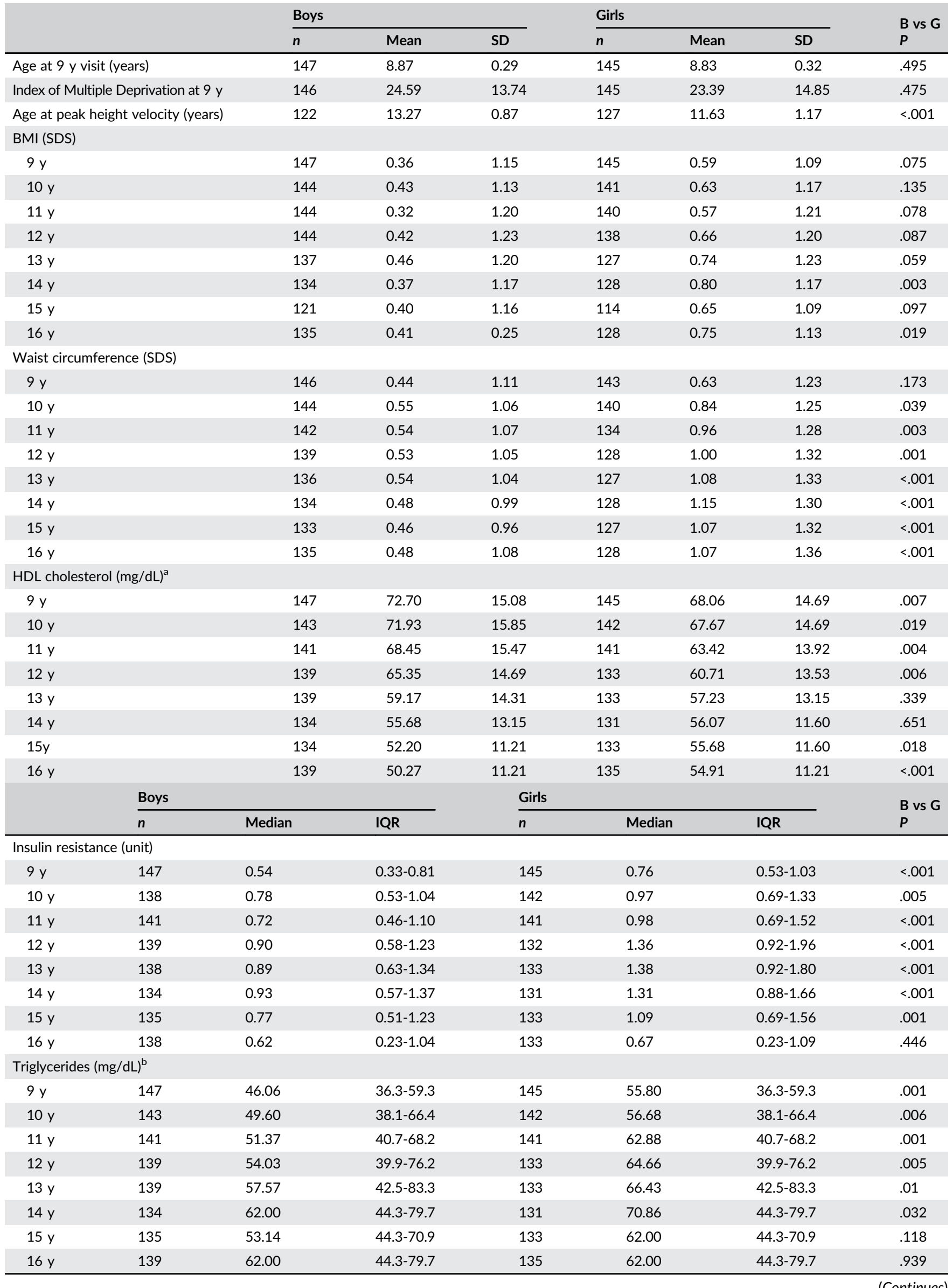


TABLE 1 (Continued)

\begin{tabular}{|c|c|c|c|c|c|c|c|}
\hline & \multicolumn{3}{|c|}{ Boys } & \multicolumn{3}{|l|}{ Girls } & \multirow{2}{*}{$\begin{array}{l}B \text { vs } G \\
P\end{array}$} \\
\hline & $n$ & Median & IQR & $n$ & Median & IQR & \\
\hline \multicolumn{8}{|c|}{ DEXA fat (\%) } \\
\hline $10 y$ & 143 & 19 & $12-27$ & 143 & 27 & $21-34$ & $<.001$ \\
\hline $11 y$ & 136 & 20 & $13-29$ & 128 & 28 & $22-36$ & $<.001$ \\
\hline $12 \mathrm{y}$ & 138 & 22 & $15-29$ & 128 & 28 & $22-36$ & $<.001$ \\
\hline $14 \mathrm{y}$ & 137 & 18 & $13-26$ & 128 & 31 & $27-38$ & $<.001$ \\
\hline $15 y$ & 117 & 16 & $12-23$ & 110 & 33 & $28-38$ & $<.001$ \\
\hline $16 y$ & 132 & 16 & $11-26$ & 126 & 34 & $29-38$ & $<.001$ \\
\hline \multicolumn{8}{|c|}{ MVPA (min/wk) } \\
\hline $9 y$ & 137 & 413 & $293-526$ & 135 & 282 & $215-362$ & $<.001$ \\
\hline $13 y$ & 123 & 380 & $248-542$ & 127 & 248 & $167-306$ & $<.001$ \\
\hline $14 y$ & 128 & 330 & $222-499$ & 115 & 205 & $151-313$ & $<.001$ \\
\hline $15 y$ & 107 & 343 & $207-463$ & 99 & 207 & $131-268$ & $<.001$ \\
\hline $16 y$ & 99 & 314 & $178-436$ & 106 & 194 & $117-285$ & $<.001$ \\
\hline
\end{tabular}

Abbreviations: B, boys; DEXA, dual energy x-ray absorptiometry; G, girls; HDL-C, high-density lipoprotein-cholesterol; IQR, interquartile range; MVPA, moderate-and-vigorous intensity physical activity; SDS, standard deviation score.

a To convert HDL-C from $\mathrm{mg} / \mathrm{dL}$ to $\mathrm{mmol} / \mathrm{L}$ divide by 38.67 .

b To convert triglycerides from $\mathrm{mg} / \mathrm{dL}$ to $\mathrm{mmol} / \mathrm{L}$ divide by 88.57 .

FIGURE 1 Insulin resistance (mean, 95\% confidence interval $[\mathrm{Cl}])$ in children from 9 to 16 years according to gender. A, Unadjusted; B, adjusted for age at peak height velocity (APHV); C, adjusted for APHV and moderate-and-vigorous intensity physical activity (MVPA); D, adjusted for APHV, MVPA, and \% fat
A

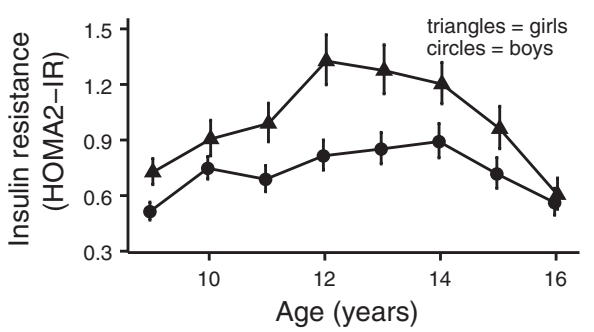

C

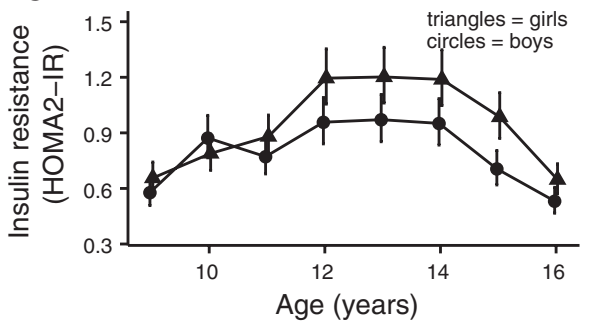

B

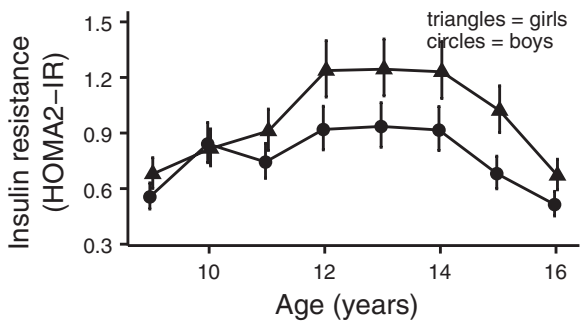

D

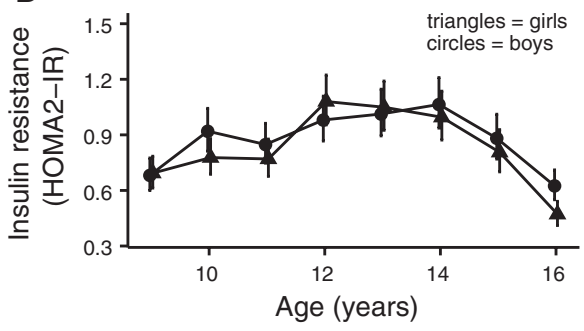

participants has allowed us to identify more precisely the age at which this gender reversal in IR occurs. The use of linear mixedeffects models allowed incorporation of all covariates and repeated measures simultaneously.

Moran et $\mathrm{al}^{14}$ did not examine the influence on IR of agedependent changes in adiposity. Changes in IR occur against a background of body fat levels that are declining in boys and increasing in girls. These changes reflect increasing lean body mass and falling body fat as boys develop adult male body composition. It appears contradictory that IR falls in girls while they continue to gain fat mass.
Despite these different patterns, the association between IR and \% fat remained stronger in girls. This may be of pathophysiological significance, with relevance to the excess risk of T2D in adolescent females.

The raw data results for triglycerides and HDL-C support the observations of Dai et $\mathrm{al}^{33}$ who studied 3 cohorts of children measured 3 times in 1 year. For both measures, girls' triglycerides were higher and HDL-C lower between 8 and 11 years, but had reversed by 14 years. Our fully adjusted trends over 8 annual time-points are able to pinpoint the ages at which this cross-over occurs. There is 
A

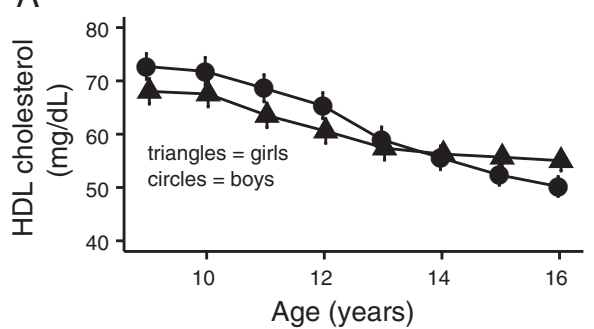

C

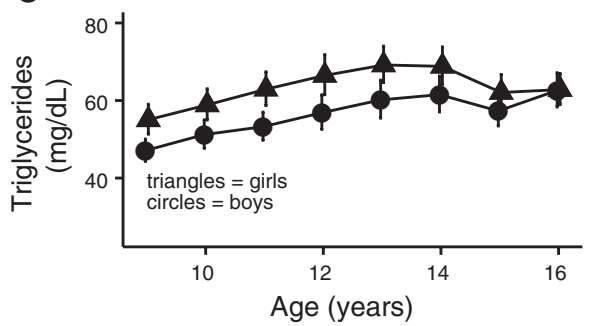

B

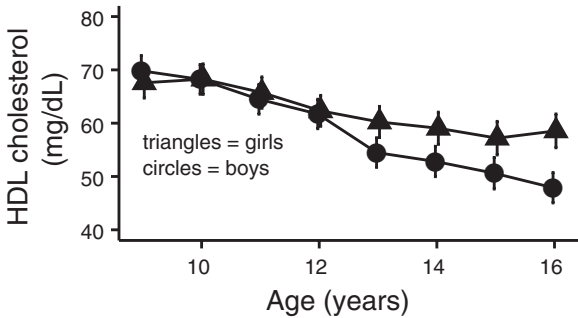

D

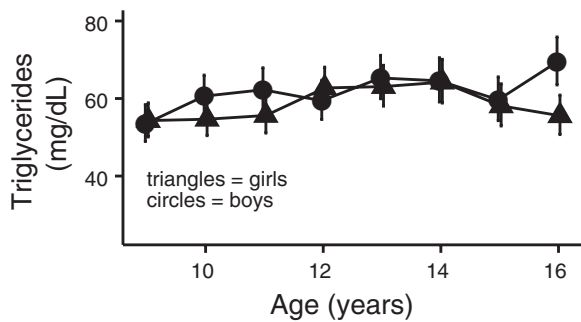

FIGURE 2 Lipid levels (mean, 95\% age at peak height velocity $[\mathrm{Cl}]$ ) in children from 9 to 16 years according to gender. A, Highdensity lipoprotein-cholesterol (HDL-C) unadjusted; B, adjusted for \% fat, age at peak height velocity (APHV) and moderateand-vigorous intensity physical activity (MVPA); C, triglycerides unadjusted; D, adjusted for \% fat, APHV, and MVPA clear divergence of HDL-C from 12 years onwards and of triglycerides from 15 years onwards. The HDL-C data are in line with the normative data collected by the Caliper study, ${ }^{34}$ showing slightly higher levels in girls from 13 to 19 years, and no gender difference in triglyceride levels.

The gender differences observed in metabolic variables could be explained by changes in fat distribution, with boys accumulating more central adiposity, known to be associated with increased IR and diabetes risk, and girls accumulating more subcutaneous and/or gluteal adiposity. However, waist circumference-SDS increased significantly in the girls from 9 to 16 years but did not change in the boys, suggesting that the girls were accumulating excess abdominal fat, while boys were growing at the expected rate. Despite this potential masking effect of excess weight gain in girls, we report a cross-over in the gender difference in IR and lipids. Therefore, we are unable to explain the observed gender differences in IR and lipids by changes in body composition. Further studies using direct measures of visceral adiposity could clarify this issue.

Another possibility is that peripubertal changes in IR are influenced by changing sex-steroid concentrations. The postpubertal decline in IR in girls might be related to rising oestrogen concentrations; oestrogen has several insulin-sensitizing effects. ${ }^{35}$ Alternatively, rising androgen levels in both genders could affect IR. Testosterone has been shown to decrease insulin sensitivity in both men and women. ${ }^{36}$ However, levels of sex hormone-binding globulin, which are strongly influenced by adiposity and IR in childhood, ${ }^{37}$ modulate the bioavailability of estradiol and testosterone so that increasing adiposity augments free concentrations of sex steroids. Therefore, while sex steroids may influence IR, there is a complex and probably bidirectional interrelationship.

Growth hormone and the growth axis are also important determinants of IR during puberty. The association plays an important physiological role, as the acceleration of growth during puberty is achieved in an energy-efficient manner mediated, at least in part, through hyperinsulinaemia/IR. ${ }^{38,39}$ The different reproductive destinies of each sex place different biological imperatives-with different tempos-on vertical growth and the acquisition of fat.
Physical activity is a potential factor that could influence metabolic health in adolescence, and may be highly relevant to diabetes and cardiovascular disease prevention strategies in young people. Physical activity declines during adolescence, ${ }^{40}$ and while there was considerable inter-individual variation in activity levels at each time point, intra-individual activity remained remarkably stable. Ranking the participants according to their 8-year activity levels allowed maximum precision, yet the overall contribution of activity to the gender difference in IR and lipids was modest.

We have previously shown that physical activity attenuates the mid-adolescent peak in IR and that this effect disappears by 16 years, independent of adiposity and pubertal stage. ${ }^{30}$ In our final model, however, physical activity explained only a small proportion of the gender difference in IR. Whether or not a modest increase in physical activity in high risk teenagers reduces their risk of T2D remains uncertain.

These findings may have practical relevance for early interventions designed to prevent or delay the onset of T2D in young people. It can be suggested that the excess risk of T2D in girls may be explained by their greater IR, although it is unclear how this is associated with beta cell failure. Weight gain between 9 and 16 years, a key influence on IR, is a potential target for intervention. Whether or not IR and other characteristics at puberty can predict subsequent hyperglycaemia will require longer term follow-up of the cohort.

This study has strengths and limitations. EarlyBird is a wellestablished longitudinal cohort study with high retention and criterion measures of IR, fatness, and activity. The narrow age range and multiple repeated measures allow for within- and between-subject comparisons. Longitudinal modelling uses all the available data while accommodating missing data points, and our methods allowed for retention of subjects in analyses despite some missing data points. Inevitably, some data is missing over an 8-year study, particularly measures of MVPA at later ages, and reliability was maximized by calculating a mean value over 8 years. Although pubertal stage was not directly assessed, APHV is a well-established objective surrogate for direct assessment of pubertal stage. It was unfeasible to take more than 1 fasting measure from the children each year, thus insulin was 
measured from a single sample rather than the mean of 3. HOMA is a surrogate measure of IR, but, correlating strongly with results from clamp studies, it is the most appropriate measure to use in young children. Accelerometry is a robust measure of physical activity, although limitations include the potential to miss upper body movement and the fact that they must be removed for water-based activities. Activity diaries reduced some of these limitations. The EarlyBird cohort is mainly Caucasian, and results may not be generalizable to populations with different ethnic composition. The current analysis terminates at 16 years when the metabolic effects of puberty may persist, especially in boys. Follow-up to adulthood will confirm whether IR remains higher in postpubertal males compared with females, before and after adjustment for adiposity, and whether IR is associated with increased long-term risk for the development of T2D.

We conclude that the higher IR of prepubertal and early pubertal girls appears to be largely explained by their greater fat mass, with more modest effects of physical activity and the timing of puberty. Around the age of 16 years a "cross-over" point is reached when, despite their lower fat mass and higher activity levels, boys become more insulin resistant than girls, and have higher triglycerides and lower HDL-C levels. The changing risk factors for boys and girls will inform diabetes prevention strategies in children.

\section{ACKNOWLEDGEMENTS}

We are sad to report that Professor Terence Wilkin died on 1 March 2017, after this paper was first submitted for publication. We acknowledge the huge contribution Terry made to diabetes research. We are indebted to the EarlyBird children and their families and would like to thank the whole research team for their contributions.

\section{REFERENCES}

1. Cali AM, Caprio S. Prediabetes and type 2 diabetes in youth: an emerging epidemic disease? Curr Opin Endocrinol Diabetes Obes. 2008;15:123-127.

2. D'Adamo E, Caprio S. Type 2 diabetes in youth: epidemiology and pathophysiology. Diabetes Care. 2011;34(suppl 2):S161-S165.

3. Ehtisham S, Hattersley AT, Dunger DB, Barrett TG. First UK survey of paediatric type 2 diabetes and MODY. Arch Dis Child. 2004;89: 526-529.

4. Dabelea D, Mayer-Davis EJ, Saydah S, et al. Prevalence of type 1 and type 2 diabetes among children and adolescents from 2001 to 2009. JAMA. 2014;311:1778-1786.

5. Dabelea D, Bell RA, D'Agostino RB Jr, et al. Incidence of diabetes in youth in the United States. JAMA. 2007;297:2716-2724.

6. Ramachandran A, Snehalatha C, Satyavani K, Sivasankari S, Vijay V. Type 2 diabetes in Asian-Indian urban children. Diabetes Care. 2003;26:1022-1025

7. Lipscombe LL, Hux JE. Trends in diabetes prevalence, incidence, and mortality in Ontario, Canada 1995-2005: a population-based study. Lancet. 2007;369:750-756.

8. Gale EA, Gillespie KM. Diabetes and gender. Diabetologia. 2001; 44:3-15

9. Cree-Green M, Triolo TM, Nadeau KJ. Etiology of insulin resistance in youth with type 2 diabetes. Curr Diab Rep. 2013;13:81-88.

10. Logue J, Walker JJ, Colhoun HM, et al. Do men develop type 2 diabetes at lower body mass indices than women? Diabetologia. 2011;54: 3003-3006.

11. Shields BM, Knight B, Hopper $\mathrm{H}$, et al. Measurement of cord insulin and insulin-related peptides suggests that girls are more insulin resistant than boys at birth. Diabetes Care. 2007;30:2661-2666.
12. Hirschler V, Maccallini G, Karam C, Gonzalez C, Aranda C. Are girls more insulin-resistant than boys? Clin Biochem. 2009;42: 1051-1056.

13. Young-Hyman D, Schlundt DG, Herman L, De Luca F, Counts D. Evaluation of the insulin resistance syndrome in 5- to 10-year-old overweight/obese African-American children. Diabetes Care. 2001;24: 1359-1364.

14. Moran A, Jacobs DR Jr, Steinberger J, et al. Changes in insulin resistance and cardiovascular risk during adolescence: establishment of differential risk in males and females. Circulation. 2008;117:23612368.

15. Hoffman RP, Vicini P, Sivitz WI, Cobelli C. Pubertal adolescent malefemale differences in insulin sensitivity and glucose effectiveness determined by the one compartment minimal model. Pediatr Res. 2000;48:384-388.

16. Murphy MJ, Metcalf BS, Voss LD, et al. Girls at five are intrinsically more insulin resistant than boys: the Programming Hypotheses Revisited-The EarlyBird Study (EarlyBird 6). Pediatrics. 2004;113: 82-86.

17. Amiel SA, Sherwin RS, Simonson DC, Lauritano AA, Tamborlane WV. Impaired insulin action in puberty. A contributing factor to poor glycemic control in adolescents with diabetes. N Engl J Med. 1986;315: 215-219.

18. Travers SH, Jeffers BW, Bloch CA, Hill JO, Eckel RH. Gender and Tanner stage differences in body composition and insulin sensitivity in early pubertal children. J Clin Endocrinol Metab. 1995;80: 172-178.

19. Geer EB, Shen W. Gender differences in insulin resistance, body composition, and energy balance. Gend Med. 2009;6(suppl 1):60-75.

20. Moran A, Jacobs DR Jr, Steinberger J, et al. Insulin resistance during puberty: results from clamp studies in 357 children. Diabetes. 1999;48:2039-2044.

21. Office for National Statistics: Ethnic group (UV09) - Neighbourhood Statistics (Plymouth, UK). 2001 Census. 2004.

22. Voss LD, Kirkby J, Metcalf BS, et al. Preventable factors in childhood that lead to insulin resistance, diabetes mellitus and the metabolic syndrome: the EarlyBird diabetes study 1. J Pediatr Endocrinol Metab. 2003;16:1211-1224.

23. Levy JC, Matthews DR, Hermans MP. Correct homeostasis model assessment (HOMA) evaluation uses the computer program. Diabetes Care. 1998;21:2191-2192.

24. Gungor N, Saad R, Janosky J, Arslanian S. Validation of surrogate estimates of insulin sensitivity and insulin secretion in children and adolescents. J Pediatr. 2004;144:47-55.

25. Cole TJ, Freeman JV, Preece MA. Body mass index reference curves for the UK, 1990. Arch Dis Child. 1995;73:25-29.

26. Tanner JM, Whitehouse RH. Clinical longitudinal standards for height, weight, height velocity, weight velocity, and stages of puberty. Arch Dis Child. 1976;51:170-179.

27. Kelly A, Winer KK, Kalkwarf $\mathrm{H}$, et al. Age-based reference ranges for annual height velocity in US children. J Clin Endocrinol Metab. 2014;99:2104-2112.

28. Metcalf BS, Curnow JS, Evans C, Voss LD, Wilkin TJ. Technical reliability of the CSA activity monitor: the EarlyBird Study. Med Sci Sports Exerc. 2002;34:1533-1537.

29. Department of Health. Start Active, Stay Active: A Report on Physical Activity for Health from the Four Home Countries' Chief Medical Officers. London, UK: Department of Health; 2011.

30. Metcalf BS, Hosking J, Henley WE, et al. Physical activity attenuates the mid-adolescent peak in insulin resistance but by late adolescence the effect is lost: a longitudinal study with annual measures from 9-16 years (EarlyBird 66). Diabetologia. 2015;58:2699-2708.

31. R Core Team (2015). R: A language and environment for statistical computing. R Foundation for Statistical Computing, Vienna, Austria. URL http://www.R-project.org/.

32. Bates $D$, Mächler $M$, Bolker $B$, Walker $S$. Fitting linear mixed-effects models using Ime4. J Stat Softw. 2015;67:48.

33. Dai S, Fulton JE, Harrist RB, Grunbaum JA, Steffen LM, Labarthe DR. Blood lipids in children: age-related patterns and association with body-fat indices: Project HeartBeat!. Am J Prev Med. 2009;37: S56-S64. 
34. Higgins V, Chan MK, Nieuwesteeg M, et al. Transference of CALIPER pediatric reference intervals to biochemical assays on the Roche cobas 6000 and the Roche Modular P. Clin Biochem. 2016;49:139-149.

35. Biundo B, Gogola M. Estradiol: the emerging evidence for a protective role against insulin resistance and obesity. Int J Pharm Compd. 2015;19:289-293.

36. Polderman KH, Gooren LJ, Asscheman H, Bakker A, Heine RJ. Induction of insulin resistance by androgens and estrogens. J Clin Endocrinol Metab. 1994;79:265-271.

37. Pinkney J, Streeter A, Hosking J, Mostazir M, Jeffery A, Wilkin T. Adiposity, chronic inflammation, and the prepubertal decline of sex hormone binding globulin in children: evidence for associations with the timing of puberty (Earlybird 58). J Clin Endocrinol Metab. 2014;99:3224-3232.

38. Arslanian SA. Clamp techniques in paediatrics: what have we learned? Horm Res. 2005;64(suppl 3):16-24.

39. Arslanian SA, Kalhan SC. Protein turnover during puberty in normal children. Am J Physiol. 1996;270:E79-E84.
40. Metcalf BS, Hosking J, Jeffery AN, Henley WE, Wilkin TJ. Exploring the adolescent fall in physical activity: a 10-yr cohort study (EarlyBird 41). Med Sci Sports Exerc. 2015;47:2084-2092.

\section{SUPPORTING INFORMATION}

Additional Supporting Information may be found online in the supporting information tab for this article.

How to cite this article: Jeffery SC, Hosking J, Jeffery AN, et al. Insulin resistance is higher in prepubertal girls but switches to become higher in boys at age 16: A Cohort Study (EarlyBird 57). Pediatr Diabetes. 2018;19:223-230. https:// doi.org/10.1111/pedi.12571 